\title{
On Modelling Reliability in RED Gateways ${ }^{\star}$
}

\author{
Vladimir V. Shakhov ${ }^{1}$, Jahwan $\mathrm{Koo}^{2}$, and Hyunseung Choo ${ }^{2, \star \star}$ \\ 1 Institute of Computational Mathematics and Mathematical Geophysics of SB RAS, \\ Prospect Akademika Lavrentjeva, 6, Novosibirsk 630090, Russia \\ shakhov@skku.edu \\ 2 School of Information and Communication Engineering, Sungkyunkwan University \\ Chunchun-dong 300, Jangan-gu, Suwon 440-746, South Korea \\ jhkoo@songgang.skku.ac.kr, choo@ece.skku.ac.kr
}

\begin{abstract}
In this paper we investigate the reliability of Random Early Detection (RED) gateway. For the RED buffer behavior a new model based on Markov chains is offered. The reliability of RED gateway is defined by an average rate of accepted packets. We examine the impact of RED tuning on the reliability by taking into account stick-slip nature of traffic intensity. The goal of the proposed model is to improve RED buffer management by using a technique of traffic intensity change detection.
\end{abstract}

\section{Introduction}

Congestion occurs on a communication link whenever the amount of traffic injected on that link exceeds its capacity. This excessive traffic causes queueing delays of packets based on buffer fill up, and in extreme cases packets are lost due to buffer overflow. To avoid such a extreme situation effectively, IETF has recommended Random Early Detection (RED) as the default queue management scheme for the next generation Internet gateways [1].

The RED mechanism is first described in [2]. The basic idea of RED is that a gateway employing RED detects congestion earlier by computing the average queue length and drops randomly the packets in buffer if the computed length remains between minimum and maximum thresholds configured manually by network administrators. Although RED has some merits points, the selection of optimal values on parameters is still an open issue according to network and traffic situation. Moreover, it is shown that a static RED cannot provide better results than tail drop in general [3. Thus, a dynamic RED mechanism is the recent focus of investigations. We present our vision of dynamic RED implementation and RED schema quality in this paper.

The remainder of the paper is organized as follows. Section 2 provides the basic notation and proposed reliability modelling approach. For the RED buffer

\footnotetext{
* This research was supported by the MIC(Ministry of Information and Communication), Korea, under the ITRC(Information Technology Research Center) support program supervised by the IITA(Institute of Information Technology Assessment), IITA-2005-(C1090-0501-0019).

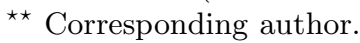


behavior a new model based on Markov chains is offered. The reliability of RED gateway is defined by an average rate of accepted packets. In Section 3 we examine the impact of RED tuning on the reliability by taking into account stick-slip nature of traffic intensity. The reason of RED gateway management modification by a point-of-change detection technique is discussed. Section 4 is a brief conclusion.

\section{RED Gateway Reliability Modelling}

Let us consider a RED scheme in detail. RED utilizes two thresholds, min threshold $h$ and max threshold $H$, and a exponentially-weighted moving average (EWMA) formula to estimate the average queue length, $Q_{\text {avg }}(t)=(1-$ $\left.W_{q}\right) Q_{\text {avg }}(t-1)+W_{q} Q$, where $Q_{\text {avg }}(t)$ is average queue length at time $t, Q$ is instantaneous queue length at time $t$ and $W_{q}$ is a weight parameter, $0 \leq W_{q} \leq 1$. A gateway implementing RED accepts all packets until the queue reaches $h$, after which it drops a packet with a probability as follows $\pi\left(Q_{a v g}\right)=\max _{p}\left(Q_{a v g}-\right.$ $h) /(H-h)$, where $\max _{p}$ is the maximal packet drop probability. When the queue length reaches $H$, all packets are dropped with a probability of one.

Let us note that a low traffic load has a little relevance to the reliability problem. The focus will be on the case of high traffic intensity. Therefore, it is reasonable to take $W_{q}=1$. Actually, if the offered load is excessive, then the probability of buffer overflow is high. Hence, we have to use an aggressive strategy of packet rejection. Assume that packet arrivals form a Poisson process with rate $\lambda$. The processing times of the packets in gateway are independent exponentially distributed random variables with mean $1 / \mu$. These are generally accepted in the literature. Packets are processed in their order of arrivals. Probability of $Q$ equals $n, n=0 \ldots H-h$ we denote by $p_{n}$.

The state diagram is given by Figure 1 where the number $Q$ is the state index. As the state diagram shows, when $Q$ exceeds the min threshold h, a part of incoming traffic should be dropped. Thus, the buffer enters state $h+i$ with probability $\alpha_{i-1}, i=2 \ldots H-h$. Let us remark $\alpha_{i}=1-\pi(h+i)$. Writing down and solving the steady-state balance equations, we get

$$
\begin{gathered}
p_{i}=\rho^{i} p_{0}, i=1 \ldots h+1 \\
p_{h+1+i}=\rho^{h+1+i} \alpha_{1} \alpha_{2} \ldots \alpha_{i} p_{0}, i=1 \ldots H-h+1,
\end{gathered}
$$

where $\rho=\lambda / \mu$ and $p_{0}=\left(\left(\rho^{h+2}-1\right) /(\rho-1)+\sum_{i=1}^{H-h-1} \rho^{h+1+i} \alpha_{1} \ldots \alpha_{i}\right)^{-1}$.

Let us now define a reliability of RED gateway. The rate of packets dropping before threshold $\mathrm{H}$ depends on $Q$. Then Average Drop Rate is calculated by

$$
A D R=\sum_{i=h+1}^{H} \pi(i) * p_{i} .
$$

Here $\pi(H)=1$, that means total-lot blocking of packets. 


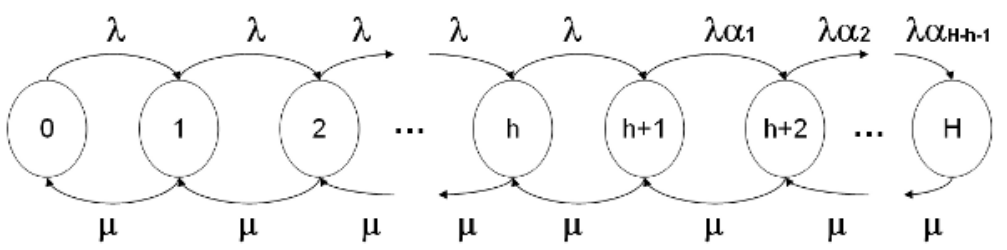

Fig. 1. Markov chain for RED gateway buffer

Actually, we have estimated an unreliability of the gateway. The packets are normally (but not always) assumed to be rejected with rate ADR. It is reasonable to define that a reliability $R$ of gateway equals $1-A D R$. It is a metric of successful packet acceptance or quality of RED schema. As we can see the considered reliability drastically depends on traffic intensity. Thus, the choice of appropriate RED parameters depends on the arrival rate.

\section{Improving the Reliability of RED Gateway}

It is often convenient to assume that the external packet traffic entering a RED gateway can be modelled by stationary stochastic process that has a constant packets arrival rate. This approximates a situation where the arrival rate changes slowly with time and constitutes what we refer to as the quasistatic assumption. When there are jumps of offered load intensity, this assumption is violated. In this case a behavior of packet arrivals remains quasistatic property on separate time durations. Using results of previous section, the optimal RED parameters can be calculated for concrete packets arrival rate. But produced RED tuning can be the worst for other packets arrival rate. In other words, if probability of gateway buffer overflow is very small, then it is not reasonable to worsen reliability (packets acceptance) by RED using. Drop Tails approach is more preferable. On the other hand, if incoming flow has got a hard rate (for example, because of DDoS attack) then protection policy should be aggressive.

Please refer to Figure 2. Calculations of reliability are made under $h=2$, $\mathrm{H}=128$. The figure shows that the RED gateway reliability degrades along with traffic load increase. It was expected. But we can see the pattern of reliability behavior is also changed. If $\rho=1.2$ then preferable $\max _{p}=0.6$. But optimal $\max _{p}$ value is differ under other $\rho$. If a change of traffic intensity is detected then use of optimal parameters $\max _{p}$ gives a reliability improvement from 0.5 percent (case of $\rho=1.1$ ) to 3.8 percent (case of $\rho=1$ ).

Thus, reliability of the RED gateway can be improved if RED parameters are recalculated for differ packets arrival rates. For this purpose, a mechanism of incoming flow intensity detection can be applied. The estimation of a moment of random process parameter changing is known as point-of-change problem (discard problem). Appropriated algorithm for discard detection under Poisson process had been proposed in [4]. 


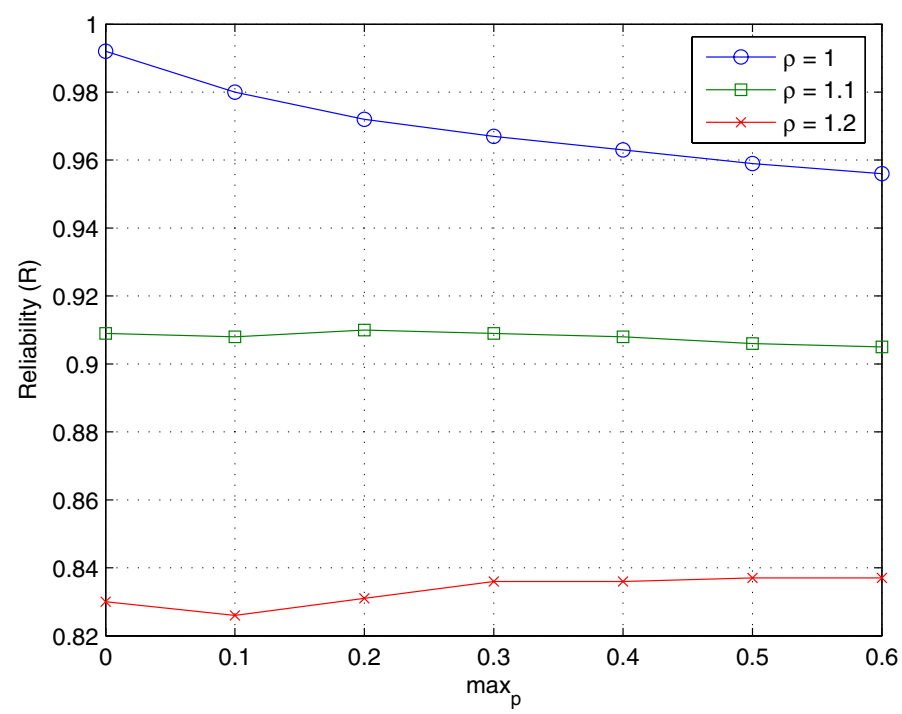

Fig. 2. Reliability behavior for RED parameter $\max _{p}$

\section{Conclusion}

On of the key network technologies is RED gateway management. For these reasons, it is important to consider the nature of RED parameters, and the manner in which it depends on the reliability of the RED gateway. In this paper the model of RED buffer behavior is offered. Its use requires simplifying assumptions but the proposed model provides a basic for adequate reliability estimations. We have shown that the stick-slip nature of traffic intensity has an essential impact on the reliability of RED gateway. Thus, dynamical tuning of RED parameters has an advantage. By this reason we offer to include point-of-change detection technique in RED gateway management.

\section{References}

1. B. Braden, et al., "Recommendations on queue management and congestion avoidance in the Internet," RFC 2309, IETF, April 1998.

2. S. Floyd and V. Jacobson, "Random early detection gateways for TCP congestion avoidance," IEEE/ACM Transactions on Networking vol. 1, no. 4, pp. 397-413, August 1993.

3. M. May, C. Diot, B. Lyles, and J. Bolot, "Reasons not to deploy RED," Seventh International Workshop on 31 May-4 June 1999, IWQoS '99, pp. 260 - 262, 1999.

4. V. Shakhov, H. Choo, Y.-C. Bang, "Discord model for detecting unexpected demands in mobile networks," Future Generation Comp. Syst. 20(2), pp. 181-188, 2004. 